\title{
The Equivalence Principle and the End of the Dark Energy
}

\author{
Joao Carlos Holland de Barcellos \\ USP: Universidade de São Paulo (Dep:SIBi), São Paulo, Brazil
}

\begin{abstract}
We will make a new approach for an effect known as "Dark Energy" by an effect on gravitatio nal field.[1] In an accelerated rocket, the dimensions of space towards movement due to 'Lorentz Contraction' are on continuous reduction.Using the equivalence principle, we presume that in the gravitational field, the same thing would happen.In this implicates in 'dark energy effect'. The calculi show that in a $7 \%$-contraction for each billion years would explain our observation of galaxies in accele rated separation.

Keywords: Dark Energy,Lorentz Contraction, Gravitation, Universe, Equivalence Principle.
\end{abstract}

\section{EQUIVALENCE PRINCIPLE AND AN ACCELERATED ROCKET}

If we think of an accelerated increasing speed rocket, its length towards movement compared to an inertial reference - will be smaller, and 'rule' within the nave will decrease continuously compared to this observer.

We would think of 'equivalence principle' to justify that gravitational field would have the same effect on 'rules' (measuring instruments) as an accelerated rocket would do within the nave, but, now, towards all gravitational field and not, in the case of rocket, only at acceleration speed.

I.e., the gravitational field would make that all rules within this field would be continuously smaller regarded to an observer outside of gravitational field and this would make, as we can see, these observers see things out of field be away fastly.

\section{LORENTZ CONTRACTION}

If we use the the Equivalence Principle we can say that gravitational field (like an acelareted rocket) contracts the space around it (including everything within). So we can explain the accelerated separation from galaxy through this contraction without postulating 'dark energy'. The contraction of space made by gravity would cause a kind of 'illusion of optic', seem like, as presented below, that galaxies depart fastly.

The contraction of space would be equivalent to relativ istic effect which occurs in a special nave in high-speed L.M.: With regard to an observer in an inertial referential stopped compared to a nave, the observer and everything is on it, including own nave, has its dimension contracted towards to movement of nave compared to a stopped observer (Lorentz Contraction).

This means that the 'rule' (measuring instruments) within the nave is smaller than the observer outside of moving nave.
The consequence is, with this 'reduced rule', this moving observer would measure things bigger than the observer would measure out of nave.

\section{THE "DARK ENERGY" THROUGH GRA VITATIONAL CONTRA CTION:}

Let's think what would happen if a light emitted by a star from a distant galaxy would arrive into our planet:

Our galaxy, as well as distant galaxies, would be in continuous contraction, as seen before, due to gravity.

A photon emitted by a star from this distant galaxy, after living its galaxy, would go through by an "empty" big space, without so much gravitational influence, until finally arrives into our galaxy and, lastly, to our planet.

During this long coursed way (sometimes billion years), this photon would suffer few gravitational effect and its wavelength would be little affected.

However, during this period, our system (our rules) would still decreasing due to gravitational field, and when this photon finally arrives here, we would measure its wavelength with a reduced 'rule' compared to what we had had at the moment when this photon was emitted from galaxy.

So, in our measurement would verify if this photon had suffered Redshift because, with reduced rule, we would measure a wavelength longer than those was measured. The traditional explanation is "Shift for Red" happened due to Doppler Effect compared to galaxy separation speed!

\section{END OF DARK ENERGY}

Farthest a galaxy is from viewpoint, more time this light will take to arrive us and more shrunken our 'rule' will be to measure this photon since it had been emitted; so it would be bigger than wavelength, which would induce us to think of faster galaxy separation speed.

This acceleration (this new explanation, only visible) from distant galaxies took astronomers to 
postulate the existence of a "Dark Energy" would have a repulsive effect, seems like they are getting away faster.

But if acceleration is due to our own scale reduction, this dark energy wouldn't be necessary anymore, because what makes this separation accelerated is, actually, our own special contraction. This would be the end of dark energy.

\section{SOME ESTIMATION}

Find below a numeral (not relativistic) estimation to estimate the contraction rate effect of our earthling system due to Redshift.

If $\mathrm{F} 0$ is the light frequency of a star which is getting away with V speed from an observer, so the frequency $\mathrm{F}$ that this observer realizes that this frequency is given by the following non-relativistic formula $(\mathrm{c}=$ light speed $)$ :

$\mathrm{F}=\mathrm{F} 0 *(1-\mathrm{V} / \mathrm{c})(1)$

But if $\mathrm{L}$ is wavelength, $\mathrm{F}$ its frequency and $c$ its speed, we have:

$\mathrm{L} * \mathrm{~F}=\mathrm{c}(2)$

If $\mathrm{L}$ is observed wavelength and $\mathrm{L} 0$ is wavelength at source, from (1) and (2), we have:

$\mathrm{L}=\mathrm{L} 0 /(1-\mathrm{V} / \mathrm{c})(3)$

Now, let's suppose that separation speed of observed galaxy follows Hubble formula (where d is the galaxy's distance from us):

$\mathrm{V}=\mathrm{H}^{*} \mathrm{~d}(4)$

So, from (3) and (4), we have:

$\mathrm{L}=\mathrm{L} 0 /\left(1-\mathrm{H}^{*} \mathrm{~d} / \mathrm{c}\right)(5)$

Now, if we detect two wavelengths L1 and L2 from two distant galaxies $\mathrm{d} 1$ and $\mathrm{d} 2$ from Earth $(\mathrm{d} 2>\mathrm{d} 1)$ which issue lights in the same wavelength L0, we can estimate the reduction on dimension rate "Fx", by an unit of time, in the date where measures were taken:

$\mathrm{Tx}=(\mathrm{L} 2-\mathrm{L} 1) / \mathrm{L} 1 / \mathrm{T}(6)$

$\mathrm{Tx}$ is reduction rate by time unit, $\mathrm{L} 2$ and $\mathrm{L} 1$ are observed wavelength and $\mathrm{T}$ is the extra time which light delays according to the second galaxy compared to the first one to arrive at our planet.

Normally the character $Z$ (redshift) [4] is designated to the factor (L2-L1)/L1:

$\mathrm{Z}=(\mathrm{L} 2-\mathrm{L} 1) / \mathrm{L} 1(7)$

$\mathrm{T}=(\mathrm{d} 2-\mathrm{d} 1) / \mathrm{c}(8)$

From (7) and (8), we have:

$\mathrm{Tx}=\mathrm{Z}^{*} \mathrm{c} /(\mathrm{d} 2-\mathrm{d} 1)(9)$

But we are using (4) and taking redshifts from each galaxy alone:

$\mathrm{Z} 1=(\mathrm{L} 1-\mathrm{L}) / \mathrm{L}$ and $\mathrm{Z} 2=(\mathrm{L} 2-\mathrm{L}) / \mathrm{L}(10)$

We have:

$\mathrm{Tx}=[(\mathrm{Z} 2-\mathrm{Z} 1) /(\mathrm{Z} 1+1)]^{*} \mathrm{H}^{*} \mathrm{c} /(\mathrm{V} 2-\mathrm{V} 1)(11)$

We can take our own galaxy as standard and simplify the formula above as redshift of our own galaxy is zero:

$\mathrm{Tx}=\mathrm{Z} * \mathrm{H} * \mathrm{c} / \mathrm{V}(12)$
Or in distances

$\mathrm{Tx}=\mathrm{Z} * \mathrm{c} / \mathrm{d}(13)$

Where:

$\mathrm{Z}$ is redshift of galaxy

$\mathrm{H}$ is Hubble constant

$\mathrm{d}$ is the galaxy's distance

$\mathrm{V}$ the apparent separation galaxy speed

$\mathrm{c}$ is light speed.

Let's use formula (13) and cosmological table data [5] for galaxy NGC3034 and calculate the real compression rate. For this galaxy:

It's interesting to note that $\mathrm{Z} / \mathrm{d}$ shall be constant which reflects the real compression rate of our coordinated system!!

$\mathrm{Tx}=0.000677 * 3 \mathrm{E} 05 /(2.72 * 3 \mathrm{E} 19)=2.5 \mathrm{E}-18 / \mathrm{s}$

To this per-second reduction rate, in one million years, the compression would be:

$\mathrm{Tx}^{*} 1$ million years $=2.5 \mathrm{E}-18 * 3 \mathrm{E} 13=0.007 \%$

I.e.: $7 \%$ of contraction every 1 billion year.

\section{REFERENCES}

[1]. Part of this theory is in the Comment at the Nature's blog: http://blogs.nature.com/news/2013/09/darkenergy-survey-launches.html 